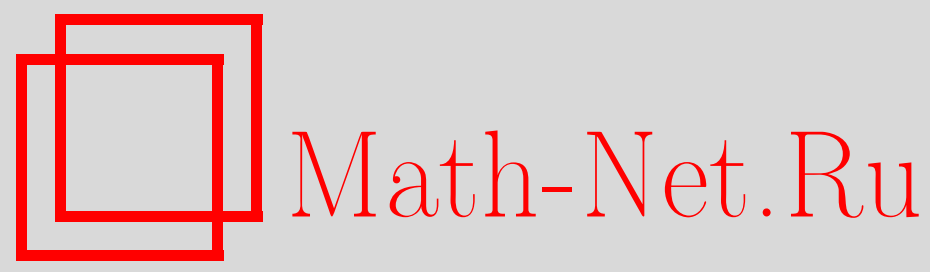

А. А. Феликсон, Кокстеровские разбиения ограниченных гиперболических пирамид и треугольных призм, Матем. заметки, 2004, том 75, выпуск 4, 624-636

DOI: https://doi.org/10.4213/mzm50

Использование Общероссийского математического портала Math-Net.Ru подразумевает, что вы прочитали и согласны с пользовательским соглашением http://www . mathnet.ru/rus/agreement

Параметры загрузки:

IP: 54.166 .219 .16

26 апреля 2023 г., $17: 47: 52$

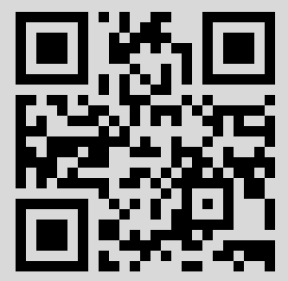




\section{КОКСТЕРОВСКИЕ РАЗБИЕНИЯ ОГРАНИЧЕННЫХ ГИПЕРБОЛИЧЕСКИХ ПИРАМИД И ТРЕУГОЛЬНЫХ ПРИЗМ}

\section{А. Феликсон}

Кокстеровским разбиением многогранника называется такое его разбиение на конечное число кокстеровских многогранников, что любые два многогранника разбиения, имеющие общую грань, симметричны друг другу относительно нее. В данной работе найдены все кокстеровские разбиения ограниченных выпуклых пирамид и треугольных призм в гиперболическом пространстве $\mathbb{H}^{3}$.

Библиография: 7 названий.

1. Введение. Рассмотрим вьпукльй многогранник $P$ в гиперболическом пространстве $\mathbb{H}^{3}$.

ОПРЕДЕЛЕНИЕ 1. Многогранник назьвается многогранником Кокстера, если все его двугранные углы являются цельми частями $\pi$.

ОПРЕДЕЛЕНИЕ 2. Кокстеровским разбиением многогранника называется такое его разбиение на конечное число кокстеровских многогранников, что любые два многогранника разбиения, имеющие общую грань, симметричны друг другу относительно этой грани.

Задача о классификации кокстеровских разбиений многогранников связана с изучением дискретных групп, порожденных отражениями. Точнее, заданный многогранник допускает кокстеровское разбиение тогда и только тогда, когда групша, порожденная отражениями относительно гиперграней данного многогранника, дискретна. Впервые аналогичная задача решена Шварцем в 1872 г. для случая сферических треугольников [1].

В гиперболическом случае задача о классификации кокстеровских разбиений далека от полного решения. Однако получены некоторые частные результаты. Кокстеровские разбиения гиперболических треугольников перечислены в 1998 г. в работе Клименко и Сакумы [2]. Разбиения гиперболических симплексов больших размерностей найдены в работах автора [3] и [4].

В данной работе найдены все кокстеровские разбиения ограниченных вьпуклых пирамид и треугольных призм в трехмерном гиперболическом пространстве $\mathbb{H}^{3}$.

Основные определения. Многогранник $P$, допускающий кокстеровское разбиение,назьвается также квази-кокстеровским. Кокстеровские многогранники разбиения из 
определения 2 называются фундаментальными многогранниками и обозначаются через $F$. Ясно, что любые два фундаментальных многогранника конгруэнтны друг другу. Плоскость $\alpha$, содержащая грань фундаментального многогранника, назьвается зеркалом, если $\alpha$ не содержит граней многогранника $P$.

В данной работе под “многогранником" мы будем понимать либо многогранник, допускающий кокстеровское разбиение, либо многогранник, ограниченньй зеркалами некоторого кокстеровского разбиения. Под "разбиением" мы будем иметь ввиду кокстеровское разбиение.

ОПРЕДЕЛЕНИЕ 3. Зафиксируем кокстеровское разбиение многогранника $P$. Для данного разбиения двугранный угол многогранника $P$, образованный гранями $\alpha$ и $\beta$, называется фундаментальным , если ни одно зеркало не содержит $\alpha \cap \beta$. При этом ребро $\alpha \cap \beta$ многогранника $P$ также называется фундаментальным. Вершина $A$ называется фундаментальной, если никакое зеркало не содержит $A$. Элементы, не являющиеся фундаментальными, называются разрезанными.

1. Фундаментальный многогранник. Пусть $P$ - квази-кокстеровский многогранник. В дальнейшем $P$ - ограниченная пирамида или ограниченная треугольная призма в $\mathbb{H}^{3}$.

Обозначим через $\alpha \cap \beta$ пересечение множеств $\alpha$ и $\beta$ во внутренней части $\mathbb{H}^{3}$.

Далее, пусть $\alpha$-грань многогранника. Обозначим через $\bar{\alpha}$ плоскость, содержащую $\alpha$.

Лемма 1. Пусть $F$ - ограниченный кокстеровский многогранник. Тогда либо $F$ является тетраэдром, либо у F есть две грани, продолэсения которых не пересекаются.

ДоКАЗАТЕЛЬСТво. Для любого многогранника без тупых углов имеем

$$
\alpha \cap \beta=\varnothing \Rightarrow \bar{\alpha} \cap \bar{\beta}=\varnothing
$$

(см. [5]). У многогранников Кокстера нет тупых углов. Поэтому достаточно доказать, что у $F$ найдутся грани $\alpha$ и $\beta$, не являющиеся смежными.

Предположим, что $\alpha \cap \beta \neq \varnothing$ для любых двух граней $\alpha$ и $\beta$ многогранника $F$. Пусть $A$ - произвольная вершина многогранника $F$. Пусть $\alpha_{1}, \ldots, \alpha_{k}-$ грани $F$, проходящие через $A$. Пусть $\beta$ - любая грань $F$, не проходящая через $A$. По предположению $\alpha_{i} \cap \beta \neq \varnothing$ для всех $i=1, \ldots, k$. Поскольку многогранник $F$ остроугольньй, у $F$ нет других граней, кроме $\alpha_{i}$ и $\beta$, т.е. $F$ - пирамида.

Предположим, что вершина $A$ не является идеальной. Тогда $A$ принадлежит ровно трем граням многогранника $F$, и $F$ является тетраэдром. Предположим, что вершина $A$ идеальна и пирамида $F$ не является тетраэдром. Рассмотрим две грани (например, $\alpha_{1}$ и $\left.\alpha_{3}\right)$, не имеющие общего ребра. Поскольку $A \notin \mathbb{H}^{3}$, получаем $\alpha_{1} \cap \alpha_{3}=\varnothing$.

Лемма 2. Пусть $P$ - ограниченная пирамида. Тогда $F-$ тетраэдр.

ДокАЗАтЕльство. Предположим, что $F$ - не тетраэдр. По лемме 1 у $F$ найдутся такие грани $\alpha$ и $\beta$, что $\bar{\alpha} \cap \bar{\beta}=\varnothing$. Пусть $F_{0}$ - фундаментальный многогранник разбиения $P$; пусть $\alpha_{0}$ и $\beta_{0}$ - непересекающиеся грани многогранника $F_{0}$. Рассмотрим последовательность фундаментальных многогранников $F_{i} \in P, i \in \mathbb{Z}$, такую, что $\alpha_{i}=\alpha_{i+1}$, если $i$ нечетно, и $\beta_{i}=\beta_{i+1}$, если $i$ четно (см. рис. 1). 


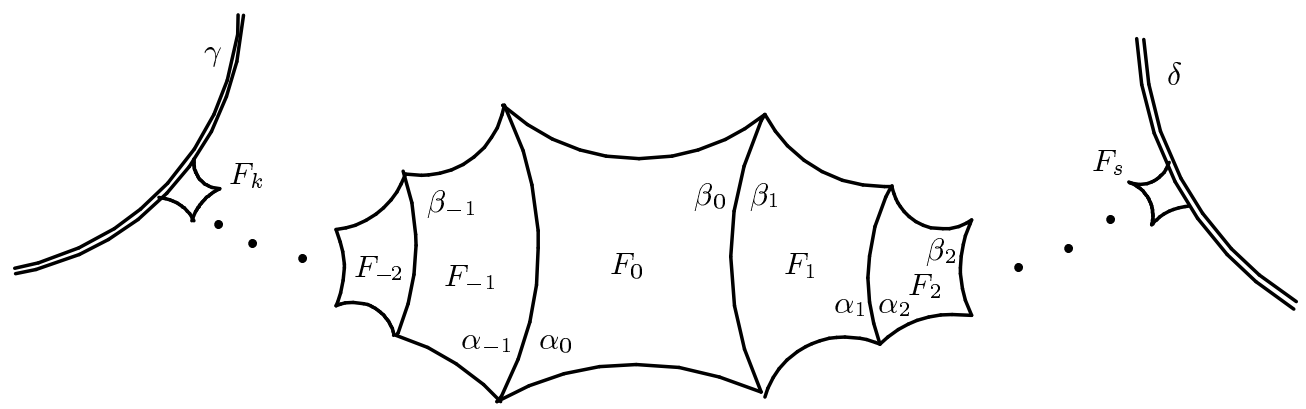

Рис. 1. Последовательность фундаментальньх многогранников

Последовательность конечна, поскольку $P$ состоит из конечного числа фундаментальных многогранников. Многогранники $F_{i}$ не образуют цикл, поскольку $\alpha_{i} \cap \beta_{i}=\varnothing$. Пусть $F_{k}$ и $F_{s}$ - первый и последний многогранники последовательности. Тогда либо $\alpha_{k}$, либо $\beta_{k}$ лежит на некоторой грани $\gamma$ многогранника $P$. Аналогично, либо $\alpha_{s}$, либо $\beta_{s}$ лежит на некоторой грани $\delta$ многогранника $P$. Ясно, что $\bar{\alpha}_{i} \cap \bar{\beta}_{j}=\varnothing$ для любых $i, j$. Но $\gamma$ пересекает $\delta$, поскольку $P$ - ограниченная пирамида. Противоречие показьвает, что $F$ - тетраэдр.

ЗАмЕчАниЕ. Идея доказательства леммы 2 принадлежит О. В. Шварцману.

Пусть $\alpha$ - зеркало кокстеровского разбиения треугольной призмы $P$. Тогда $\alpha$ пересекает $P$ одним из способов, представленных на рис. 2.1-2.16.

1)

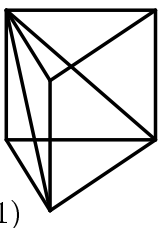

2)

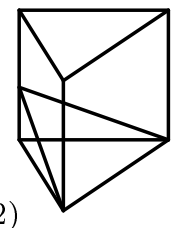

3)

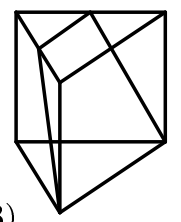

4)

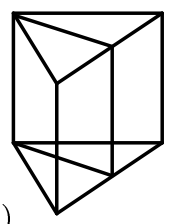

5)

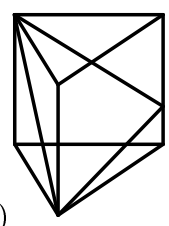

6)

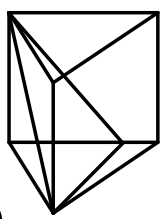

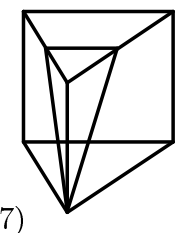

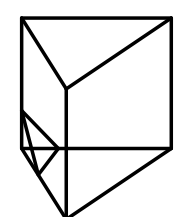

12)

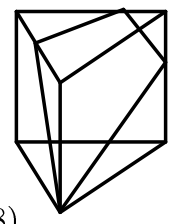

8)
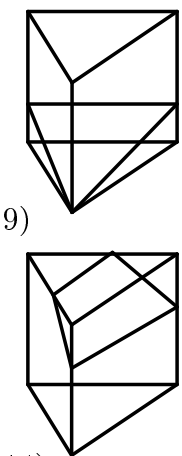

14)
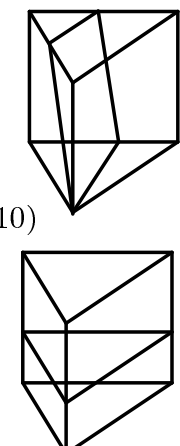

15)

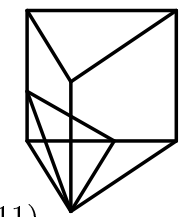

11)

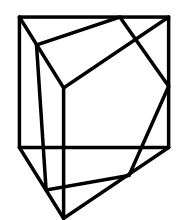

16)

Рис. 2. Зеркала в призме

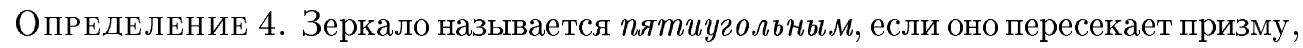
как показано на рис. 2.16. 
ОПРеДЕЛЕниЕ 5. Треугольная квази-кокстеровская призма $P$ назьвается минимальной, если любая призма, лежащая внутри $P$, фундаментальна.

Лемма 3. Пусть $P$ - минимальная призма и $F-$ фундаментальный многогранник разбиения. Пусть многогранник $F$ отличен от тетраэдра и треугольной призмы. Тогда любое зеркало разбиения является пятиугольным и все двугранные угль призмы $Р$ фундаментальны.

ДокАЗАтЕльСтво. Предположим, что $P$ содержит тетраэдр или пирамиду. Тогда по лемме $2 F$ - тетраэдр. Поэтому $P$ не содержит ни тетраэдра, ни пирамиды. Поскольку призма $P$ минимальна и $F$ не является треугольной призмой, $P$ не содержит меньших треугольных призм. Поэтому любое зеркало является пятиугольньг (см. рис. 2). Ясно, что при этом все двугранные углы призмы $P$ фундаментальны.

Лемма 4. Пусть $P$ - треугольная призма. Тогда F является либо тетраэдром, либо треугольной призмой.

ДокАЗАТЕЛьСтво. Достаточно доказать лемму для минимальной призмы $P$. Предположим, что многогранник $F$ отличен от тетраэдра и треугольной призмы. Тогда по лемме 3 все зеркала являются пятиугольньми и все двугранные углы призмы $P$ фундаментальны.

Пусть $A B C D E$ - произвольное пятиугольное зеркало (см. рис. 3). Это зеркало отделяет точки $M_{1}, N_{1}, N_{3}$ от точек $M_{2}, M_{3}, N_{2}$.

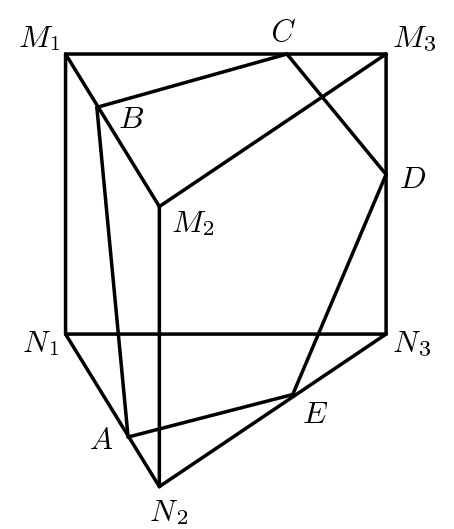

Рис. 3. Пятиугольное зеркало

Многогранник $W$ будем называть хорошим, если $W$ содержит точки $M_{2}, M_{3}, N_{2}$ и если его можно отрезать от $P$ одним зеркалом. Пусть $W$ - минимальньй хороший многогранник (т.е. $W$ не содержит хороших многогранников). Такой минимальньй многогранник существует, поскольку разбиение содержит конечное число зеркал. Пусть $W-$ многогранник с вершинами $M_{2} M_{3} N_{2} A B C D E$ (см. рис. 3 ).

Предположим, что у $W$ есть разрезанньй двугранньй угол $\alpha$. По лемме 3 угол $\alpha$ образован плоскостью $A B C D E$ и одной из граней призмы $P$. Пусть П - зеркало, разрезающее угол $\alpha$. Ясно, что точки $M_{1}, N_{1}$ и $N_{3}$ лежат по одну сторону от П. Поскольку 
зеркало П пятиугольное, П отделяет точки $M_{1}, N_{1}$ и $N_{3}$ от точек $M_{2}, M_{3}$ и $N_{2}$. Поэтому П отрезает от $P$ хороший многогранник, лежащий внутри $W$. Это противоречит минимальности многогранника $W$.

Таким образом, все двугранные углы многогранника $W$ фундаментальны, и следовательно, $W$ является многогранником Кокстера. Продолжения непересекающихся граней многогранника Кокстера не могут пересекаться (см. [5]). В случае многогранника $W$ это свойство не выполнено: продолжения граней $A E N_{2}$ и $C D M_{3}$ пересекаются по прямой $N_{1} N_{3}$. Противоречие показьвает, что $F$ - либо тетраэдр, либо треугольная призма.

2. Разбиения пирамид. В данном пункте мы опишем алгоритм, позволяюший перечислить все кокстеровские разбиения ограниченных гиперболических пирамид.

Пусть $P$ - пирамида, имеющая только пять вершин: $O, A_{1}, A_{2}, A_{3}, A_{4}$ (где $A_{1} A_{2} A_{3} A_{4}$ - основание и $O$ - вершина). По лемме $2 F$ является тетраэдром. Предположим, что все ребра вида $O A_{i}$ фундаментальны. Рассмотрим сферу $s$ небольшого радиуса с центром в точке $O$. Пересечение $s \cap P$ является сферическим четырехугольником $q$, все углы которого фундаментальны. Это невозможно, поскольку сумма углов сферического четырехугольника должна быть больше, чем $2 \pi$.

Поэтому можно считать, что некоторое зеркало $m$ проходит через ребро $O A_{1}$. Это зеркало разрезает пирамиду на двеменьших пирамиды. Одна из этих пирамид является тетраэдром, а другая - либо тетраэдром, либо четырехугольной пирамидой. Таким образом, минимальная пирамида состоит из двух тетраэдров (возможно, не являющихся фундаментальными). Кокстеровские разбиения гиперболических тетраэдров перечислены в [3]. Поэтому можно найти разбиения минимальных четырехугольных пирамид. Зная разбиения минимальных пирамид, можно перечислить и разбиения бо́льших пирамид. Таким образом, переходя от меньших четырехугольных пирамид к бо́льшим, можно найти разбиения всех четырехугольньх пирамид.

Аналогично, можно перечислить разбиения пирамид с бо́льшим числом вершин. Каждая пирамида $O A_{1} \ldots A_{n}$ имеет разрезанное ребро $O A_{i}$. Таким образом, каждая пирамида разрезана на две меньших пирамиды, все возможные разбиения которых должны быть перечислены на предыдущих шагах.

Данный алгоритм, реализованньй на компьютере, приводит к большому списку четырехугольных квази-кокстеровских пирамид, нескольким пятиугольньм квази-кокстеровским пирамидам и ровно одной шестиугольной квази-кокстеровской пирамиде. Список этих пирамид можно найти в [6].

3. Разбиения призм на призмы. В данном пункте $P$ и $F$ - треугольные призмы.

ЛЕмма 5. Ни одно основание ни одной фундаментальной призмы не лехит на боковой грани призмы $P$.

ДокАЗАТЕЛЬСТво. Пусть основание фундаментальной призмы $F_{1}$ содержится в боковой грани $s$ призмы $P$. Рассмотрим последовательность всех фундаментальных призм

$$
F_{1}, F_{2}, \ldots, F_{n}, \ldots, \quad F_{i} \in P
$$

таких, что призмы $F_{i}$ и $F_{i+1}$ имеют общее основание. Число различных членов этой последовательности конечно, поскольку $P$ содержит конечное число фундаментальных 
призм. Плоскости, содержащие основания призмы $F_{1}$, расходятся, так как $F_{1}-$ призма Кокстера. Следовательно, все плоскости, содержащие основания призм $F_{i}$, попарно расходятся. Поэтому последовательность призм не может зациклиться и в ней есть последняя призма $F_{n}$. Одно из оснований призмы $F_{n}$ содержится в какой-то грани $b$ призмы $P$ (иначе последовательность можно было бы продолжить). Как основания фундаментальных призм, грани $s$ и $b$ расходятся. Это противоречит тому, что $s$, как боковая грань призмы $P$, пересекается со всеми гранями призмы $P$.

ЛЕмма 6. Ни одна боковая грань ни одной фундаментальной призмы не лежит на основании призмы $P$.

ДокАЗАТЕЛЬСтво. Обозначим через $b$ и $s$ основания призмы $P$. Пусть боковая грань $a_{0}$ фундаментальной призмы $F_{0}$ лежит на основании $b$ призмы $P$. Рассмотрим последовательность всех фундаментальных призм

$$
F_{-k}, \ldots, F_{0}, \ldots, F_{l}, \quad F_{i} \in P
$$

таких, что $F_{i}$ и $F_{i+1}$ имеют общее основание. Последовательность конечна и не может зациклиться. Значит, основания $a_{-k}$ и $b_{l}$ призм $F_{-k}$ и $F_{l}$ лежат на гранях призмы $P$. По лемме 5 основания $a_{-k}$ и $b_{l}$ лежат на двух разных основаниях призмы $P$. Пусть $a_{-k}$ лежит на основании $s$. Тогда $a_{-k}$ пересекает $a_{0}$ (см. рис. 4 ). Но это невозможно, поскольку грани $a_{-k}$ и $a_{0}$ должны расходиться.

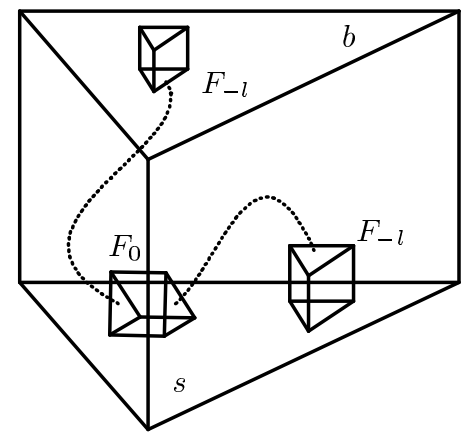

Рис. 4. Боковая сторона призмы $F$ не лежит на основании призмы $P$

ЛЕмма 7. Пусть $b$-основание призмы $P, a b_{1} u b_{2}$ - основания фундаментальной треугольной призмы $F$. Пусть грань $b$ не фундаментальна. Тогда замощение грани $b$ гранями призмы $F$ является кокстеровским разбиением. При этом фундаментальным треугольником является треугольник $b_{1}$ или $b_{2}$.

ДокАЗАТЕльство. По лемме 6 грань $b$ замощена треугольниками. Соседние ячейки получившегося замощения являются гранями призмы $F$, имеющими общее ребро. По предыдущей лемме все эти ячейки являются треугольньпи гранями. Пусть одна из ячеек является основанием $b_{1}$. Тогда и все остальные - тоже образы этого основания. Очевидно, что такое замощение является кокстеровским разбиением.

Из леммы 5 следует, что любая боковая грань призмы $P$ замощена боковыми гранями призмы $F$. Эти боковые грани будем называть ячейками замощения грани $s$. Эти 
замошения не обязательно являются кокстеровскими разбиениями. Тем не менее, следующие две леммы показьвают, что такие замощения устроены достаточно просто.

Лемма 8. Пусть $s-$ боковая грань призмы $P$. Тогда зеркала кокстеровского разбиения призмы $P$ высекают на s “решетку" (см. рис. 5а). То есть

1) любая вершина грани s принадлежит ровно одной ячейке;

2) любая внутренняя точка стороны четырехугольника $s$ принадлежит ровно одной или двум ячейкам;

3) любая внутренняя точка грани s принадлежит одной, двум или четырем ячейкам.

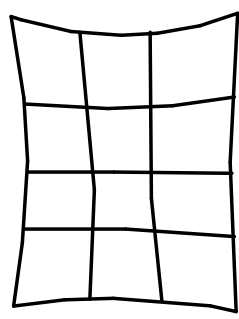

a)

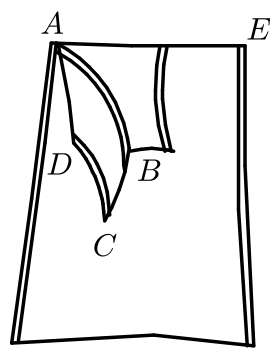

b)

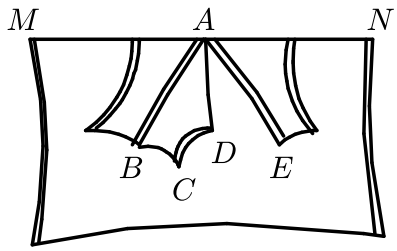

c)

Рис. 5. Замощение боковой грани напоминает решетку.

ДокАЗАТЕЛЬСТво. Сторону $A B$ ячейки назовем горизонтальной, если ребро $A B$ принадлежит основанию фундаментальной призмы; в противном случае, сторону $A B$ назовем вертикальной. Рассмотрим произвольную ячейку $A B C D$; пусть $A B$ и $C D$ - ее вертикальные стороны. Построим такую последовательность ячеек, содержащую $A B C D$, что любые две соседние ячейки имеют общую горизонтальную сторону. Такую последовательность будем назьвать вертикальной. Аналогично, можно построить горизонтальную последовательность, в которой соседние ячейки имеют общую вертикальную сторону. Очевидно, вертикальная последовательность кончается на двух различных горизонтальных сторонах грани $s$, а горизонтальная - на двух различных вертикальных сторонах. Покажем, что это условие не вьполнено, если утверждение леммы не верно.

1) Пусть вершина $A$ грани $s$ принадлежит двум или более ячейкам (см. рис. 5b). Рассмотрим вертикальную последовательность, построенную по ячейке $A B C D$. Все ячейки этой последовательности лежат с одной стороны от прямой $A B$. Поэтому последовательность не может кончаться на стороне $A E$, и мы получаем условие 1$)$.

2) Пусть точка $A$ лежит на горизонтальной стороне $M N$ грани $a$. Пусть $A$ принадлежит трем или более ячейкам. Тогда среди них есть ячейка $A B C D$, ни одна сторона которой не лежит на $M N$ (см. рис. $5 c$ ). Точка $A$ принадлежит как минимум двум вертикальным сторонам ячеек. Рассмотрим вертикальную последовательность, построенную по ячейке $A B C D$. Очевидно, все ячейки этой последовательности лежат внутри угла $\angle B A E$. Поэтому последовательность не может кончаться на стороне $M N$.

Аналогичное рассуждение применимо в случае точек вертикальных сторон, и мы получаем 2 ). 
3) Пусть $A$ лежит внутри четырехугольника $s$. Ясно, что $A$ не может принадлежать ровно трем ячейкам. Пусть $A$ принадлежит пяти или более ячейкам.

Пусть $a_{1}$ - произвольная ячейка и $a_{1}, \ldots, a_{k}$ - часть вертикальной последовательности, кончающаяся на горизонтальной стороне. Тогда будем говорить, что ячейка лежит в $k$-ом слое. Точку $X$ назовем точкой $k$-го слоя, если $X$ лежит в ячейке $k$-го слоя. Если точка принадлежит нескольким ячейкам, определим слой этой точки как максимально возможньй.

В части 2) данного доказательства лемма доказана для ячеек первого слоя. Аналогичные рассуждения показьвают, что если лемма верна для точек $k$-го слоя, то она верна и для точек $(k+1)$-го слоя. Тогда при помощи рассуждений из п. 2) доказательства данной леммы можно показать, что утверждение верно для ячеек $(k+1)$-го слоя. Таким образом, утверждение верно для всех слоев.

ЛЕмма 9. Боковые грани призмы Р разрезаны одним из способов, представленных на рис. 6.

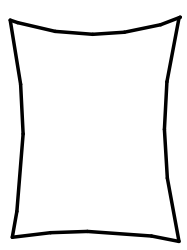

a)

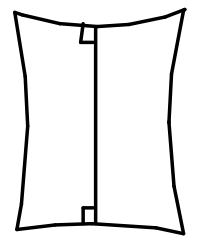

b)

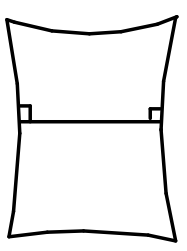

c)

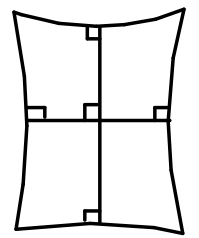

d)

Рис. 6. Замощения боковых граней

ДокАЗАТЕЛЬСтво. Пусть $s$ - боковая грань призмы $P$. Поскольку призма $F$ является кокстеровским многогранником, все ее грани остроугольные. Поэтому "прутья" решетки пересекают друг друга и границу четырехугольника $s$ под прямыми углами. Две расходящиеся прямые на плоскости Лобачевского имеют только один общий перпендикуляр. Поэтому не может быть двух горизонтальных или вертикальных прутьев.

Лемма 10. Основания призмы Р разрезаны одним из способов, представленных на puc. 7.

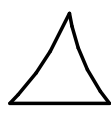

$(k, l, m)$

a)

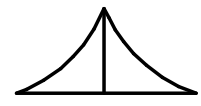

$(2, k, l)$

b)

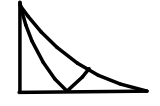

$(2,3, k)$

c)

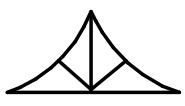

$(2,4, k)$

d)

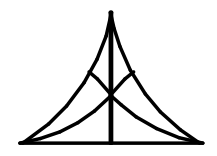

$(2,3, k)$

e)

Рис. 7. Разбиения оснований (под каждым из разбиений указаны углы фундаментального треугольника) 
ДокАЗАТЕЛЬСТво. Пусть $s$ - основание призмы $P$. По лемме 7 основание $s$ либо фундаментально, либо допускает кокстеровское разбиение. Кокстеровские разбиения треугольников найдены в [2]. Из леммы 9 следует, что ни одна сторона треугольника $s$ не может быть разрезана более, чем на две части. Этому условию удовлетворяют только разбиения, указанные на рис. 7.

ТЕОрема 1. Пусть треугольная призма $P$ допускает кокстеровское разбиение и $F$ является треугольной призмой. Тогда разбиение призмы $P$ является одним из разбиений, перечисленных на рис. 8.

ДокАЗАТЕЛЬСТво. Из леммы 8 следует, что оба основания разрезаны одинаково. По лемме 10 основания призмы разрезаны одним из пяти способов. Рассмотрим два случая.

1) Пусть основания не разрезаны. Тогда боковые грани либо фундаментальны, либо разрезаны, как на рис. 6(c). Очевидно, все три боковые грани разрезаны или неразрезаны одновременно; т.е. единственньм возможным кокстеровским разбиением, в котором основания неразрезаны, является разбиение, показанное на рис. 8 сверху (никаких других зеркал быть не может, поскольку каждое зеркало разрезает границу призмы $P$ ).

2) Пусть основания разрезаны. По виду оснований получаем два возможных вида боковых граней: с горизонтальным разрезом или без него. Отсюда получаем два вида разбиений призмы. При этом однозначно определяется часть двугранных углов фундаментальной призмы $F$. Остается проследить за тем, чтобы оставшимся двугранным углам можно было присвоить такие значения, чтобы были вьполнены условия теоремы Андреева (см. [7]). Если основания разрезаны, как на рис. 7b или 7c, то найти такие углы можно. В случаях $7 \mathrm{~d}$ и 7 е этого сделать нельзя.

4. Разбиения призм на тетраэдры. В данном пункте $P$ - треугольная призма, а $F-$ тетраэдр.

Каждая грань призмы $P$ замощена треугольниками, но в большинстве случаев эти замощения не являются кокстеровскими разбиениями. Изучим, как может вьглядеть разбиение треугольных оснований призмы.

ОПРЕДЕЛЕНИЕ 6. Пусть двугранньй угол при ребре фундаментального тетраэдра

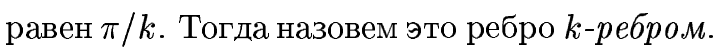

Лемма 11. Пусть все плоские углы основания $p$ призмы $P$ неразрезаны. Тогда р состоит из одного треугольника. Если эме один угол треугольника р разрезан двумя зеркалами на три части, то треугольник р ограничен 2-ребром, 3-ребром и 5-ребром.

ДокАЗАТЕльство. Пусть $f$ - грань фундаментального тетраэдра $F$, а $\bar{f}$ - плоскость, содержащая $f$. Ясно, что плоскость $\bar{f}$ замощена треугольниками, равными граням тетраэдра $F$. Несложно восстановить это замощение. В самом деле, рассмотрим грань $f$ и найдем ее непосредственных соседей на плоскости $\bar{f}$. В зависимости от двугранных углов это будут либо копии этой жеграни, либо копии других граней тетраэдра (если двугранньй угол равен $\pi / k$, где $k$ четно, то получится эта же грань, в противном случае - соседняя). Продолжая приклеивать треугольники один за другим, получим 

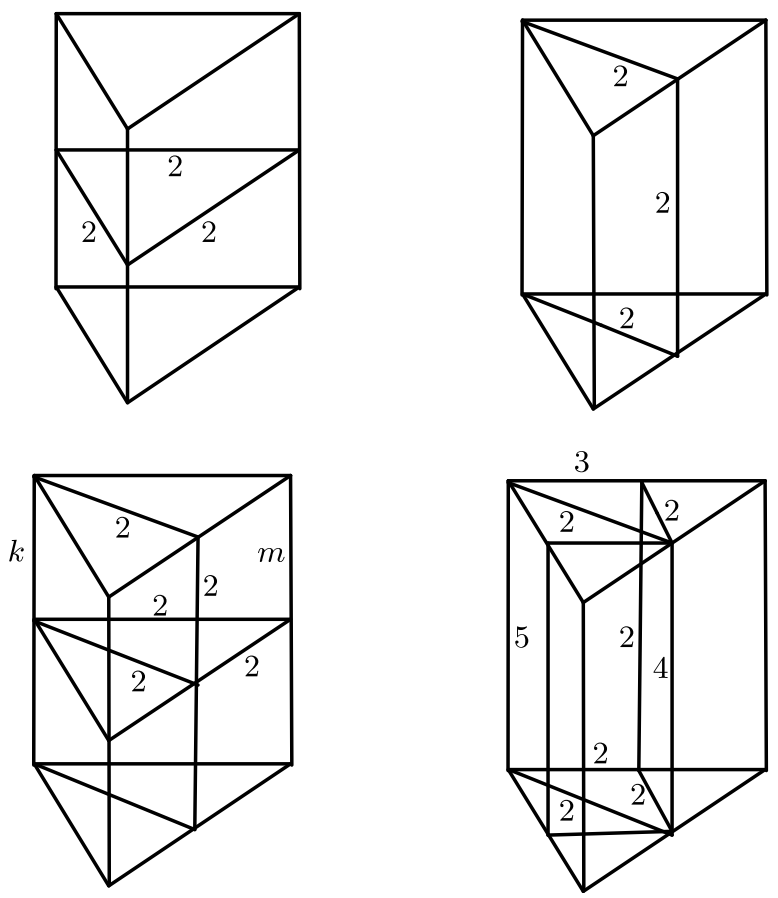

$k, m=4$ или 5

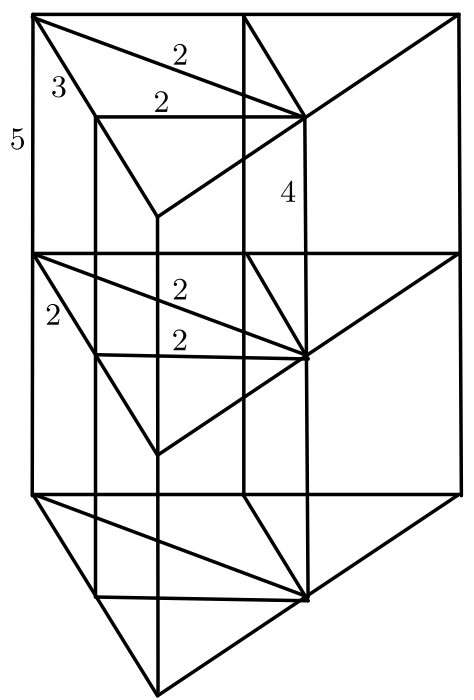

Рис. 8. Разбиения треугольных призм на призмы

разбиение в сколь угодно большой окрестности треугольника $f$. Единственный вопрос состоит в том, когда треугольники замкнутся вокруг вершины.

Это тоже нетрудно выяснить. Пусть $A$ - вершина фундаментального тетраэдра, в которой сходятся $k$-ребро, $l$-ребро и $m$-ребро. Пусть $A$ лежит в плоскости $\bar{f}$. Тогда разбиение маленькой трехмерной сферической окрестности точки $A$ соответствует 
кокстеровскому разбиению сферы с фундаментальным треугольником $(\pi / k, \pi / l, \pi / m)$. Пересечение этой окрестности с плоскостью $\bar{f}$ соответствует (сферической) прямой на разбиении сферы. Чтобы узнать, сколько лучей, лежащих в $\bar{f}$, выходит из точки $A$, достаточно сосчитать, сколько вершин треугольников лежит на соответствующей сферической прямой.

Итак, для каждой грани каждого кокстеровского тетраэдра мы можем построить соответствующее разбиение плоскости. Рассмотрев эти разбиения, несложно увидеть, что любой треугольник, углы которого неразрезаны, является гранью фундаментального тетраэдра $F$. Это доказьвает первое утверждение леммы. Кроме того, можно заметить, что треугольник с углом, разрезанным на три части, есть только в одном из этих разбиений. Такой треугольник замощен, как показано на рис. 9, и ограничен 2-ребром, 3-ребром и 5-ребром.

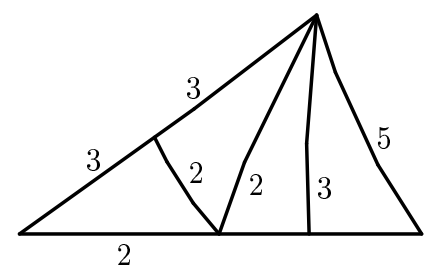

Рис. 9. $k$-ребра отмечены меткой $k, k=2,3,5$

Лемма 12. Пусть вериина А призмы Р разрезана. Тогда разрезан хотя бы один из двугранных углов при ребрах, содержащих $A$.

ДокАЗАТЕЛЬСтво. Допустим, что все двугранные углы при ребрах, содержащих $A$, фундаментальны. Рассмотрим маленькую сферу $s$ с центром в точке $A$. Разбиение призмы $P$ высекает на $s$ кокстеровское разбиение сферического треугольника $p=s \cap P$. Ясно, что все углы треугольника $p$ также фундаментальны. Легко проверить, что при этих условиях треугольник $p$ допускает разбиение, представленное на рис. 10. Все углы этого сферического треугольника прямые.

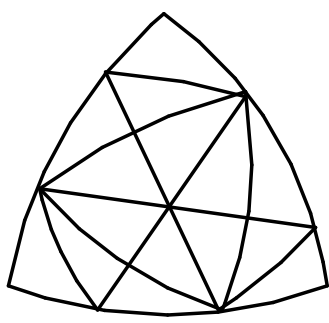

a)

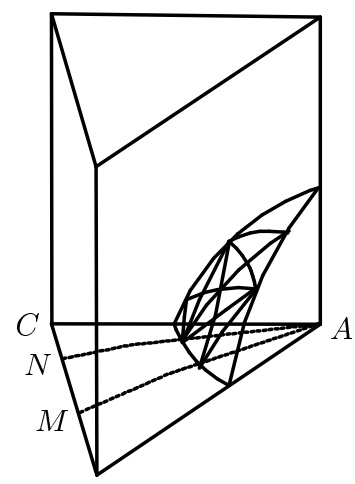

$B$

b)

Рис. 10. Если вершина $A$ призмы $P$ разрезана, но все ребра, кончающиеся в $A$, фундаментальны, то $A B$ и $A C$ являются 2-ребрами, и разбиение $\triangle A B C$ невозможно 
Таким образом, вершина $A$ разрезана, как показано на рис. $10(\mathrm{~b})$. При этом каждое ребро призмы, содержащее вершину $A$, является 2-ребром. Рассмотрим нижнее основание призмы. Это треугольник $A B C$, угол $A$ которого разрезан на три части зеркалами $A N$ и $A M$. По лемме 11 этот треугольник должен быть ограничен 2-ребром, 3 -ребром и 5-ребром. Это противоречит тому, что $A B$ и $A C$ являются 2-ребрами.

Лемма 13. Треугольная призма, допускающая разбиение на тетраәдрь, имеет разрезанный двугранный угол.

ДокАЗАТЕЛЬСТВо. Предположим, что все двугранные углы призмы неразрезаны. Тогда по лемме 12 неразрезаны и все вершины призмы, и из леммы 11 следует, что каждое основание состоит ровно из одной грани тетраэдра $F$. Рассмотрим фундаментальный тетраэдр $F_{0}$, содержащий основание $\alpha$. Поскольку двугранные углы неразрезаны, боковые грани призмы вместе с основанием $\alpha$ составляют все грани тетраэдра $F_{0}$, что невозможно.

Алгоритм поиска разбиений призм на тетраэдры. Итак, мы доказали, что любая призма имеет разрезанный двугранный угол. Поэтому из рис. 2.1-2.4 ясно, что любая треугольная призма состоит из двух меньших призм, из меньшей призмы и тетраэдра, или из тетраэдра и четырехугольной пирамиды. Зная разбиения меньших частей, мы сможем восстановить разбиение всей призмы.

Напомним, что призма назьвается минимальной, если она не фундаментальна и не содержит меньших нефундаментальньх призм.

ОПРЕДЕЛЕНИЕ 7. Минимальная призма назьвается призмой нулевого уровня. Призму $P$, содержащую меньшую призму, назовем призмой уровня $k+1$, если $P$ содержит призму уровня $k$, но ни одна призма, лежащая в $P$ не содержит призмы уровня $k$.

Найдем сначала все минимальные разбиения призмы. Ясно, что в минимальной призме не может быть зеркал, изображенных на рис. 2.2-2.4. Поэтому есть зеркало вида рис. 2.1 , например, зеркало $A_{1} B_{2} B_{3}$ на рис. 11 . Тогда вершина $A_{1}$ разрезана, и из леммы 12 следует, что существует зеркало, содержащее ребро с концом в $A_{1}$. Это зеркало не может содержать $A_{1} B_{1}$, поскольку призма минимальна. Таким образом, оно содержит либо $A_{1} A_{2}$, либо $A_{1} A_{3}$ (можно считать, что $A_{1} A_{2}$, см. рис. 10). Таким образом, мы разбили призму на три тетраэдра. Все кокстеровские разбиения тетраэдров известны из [3]. Поэтому можно найти все разбиения минимальных призм.

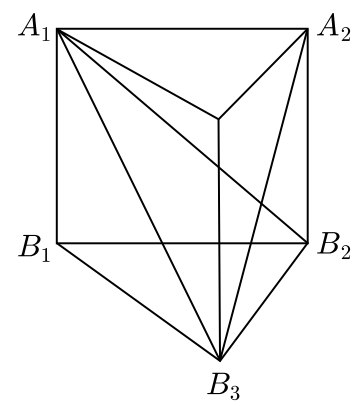

Рис. 11. Минимальная призма состоит из трех тетраэдров, возможно не фундаментальных 
Предположим, что мы нашли все разбиения призм уровня $k$, а такжевсех призм меньшего уровня. Найдем все разбиения призм уровня $k+1$. Допустим, что у призмы $P$ есть двугранный угол, разрезанный зеркалом как на рис. 2.2-2.4. По определению, каждая из частей, являющихся треугольной призмой, является призмой уровня не вьше $k$. Все возможные разбиения таких призм мы уже перечислили. Разбиения тетраэдров нам тоже известны. Таким образом, в этом случае мы можем восстановить разбиение призмы $P$. Допустим теперь, что таких зеркал, как на рис. $2.2-2.4$, в разбиении призмы $P$ нет. Тогда в этом разбиении есть зеркало вида рис. 2.1. Причем, как и в случае минимальных призм, таких зеркал как минимум два, и расположены эти два зеркала так, что призма $P$ разбивается ими на три тетраэдра (мы снова использовали лемму 12 и отсутствие зеркал с рис. 2.2-2.4). Все возможные разбиения тетраэдров мы знаем, поэтому можем перечислить все возможные разбиения призмы $P$ и в этом случае.

Итак, используя этот алгоритм, можно перечислить все призмы уровня не выше, чем любое целое число. При выполнении этой процедуры выясняется, что ни одной призмы уровня восемь нет. Поэтому нет и ни одной призмы большего уровня. Таким образом, можно перечислить все разбиения треугольных призм. Алгоритм реализован на компьютере, в результате чего получено 73 разбиения треугольных призм на тетраэдры. Таблица с описанием этих разбиений приведена в [6].

\section{СПИСОК ЦИТИРОВАННОЙ ЛИТЕРАТУРЫ}

[1] Schwarz H. A Über diejenige Fälle in welchen die Gaussische hypergeometrische Reihe eine algeraische Function ihres viertes elementes darstellt // Crelle's J. 1873. V. 75. P. 292-335.

[2] Klimenko E., Sakuma M. Two-generator discrete subgroups of Isom( $\left.\mathbb{H}^{2}\right)$ containing orientation-reversing elements // Geom. Dedicata. 1998. V. 72. P. 247-282.

[3] Felikson A. Coxeter decompositions of hyperbolic tetrahedra // E-print math.MG/0212010, 2002.

[4] Феликсон А. Кокстеровские разбиения гиперболических симплексов // Матем. сб. 2002. T. 193. №12. C. 134-156.

[5] Андреев Е.M. О пересечении плоскостей граней многогранников с острыми углами // Матем. заметки. 1970. Т. 8. №4. С. 521-527.

[6] Felikson A. Coxeter decompositions of hyperbolic pyramids and triangular prisms // E-print math.MG/0212195, 2002.

[7] Андреев Е. М. О выпуклых многогранниках в пространствах Лобачевского // Матем. сб. 1970. T. 81. № 3. C. 445-478.

Независимый Московский университет

Поступило

E-mail : felikson@mccme.ru

15.01 .2003

Исправленный вариант

19.05.2003 